\section{OP0123 EVALUATION OF RETINAL MICROVASCULATURE BY OPTICAL COHERENCE TOMOGRAPHIC ANGIOGRAPHY IN SYSTEMIC LUPUS ERYTHEMATOSUS PATIENTS MIRRORS SYSTEMIC INVOLVEMENT}

P. Conigliaro ${ }^{1}$, M. Cesareo $^{2}$, C. Canofari ${ }^{1}$, G. Aloe $^{2}$, G. Alvaro $^{2}$, P. Triggianese ${ }^{1}$ A.G. Salandri ${ }^{2}$, M.S. Chimenti ${ }^{1}$, R. Perricone ${ }^{1} .{ }^{1}$ Rheumatology, Allergology and Clinical Immunology Clinic, Department of 'Medicina dei Sistemi'; ${ }^{2}$ Ophthalmology Unit, Department of Experimental Medicine and Surgery, University of Rome Tor Vergata, Rome, Italy

Background: Optical coherence tomographic angiography (OCTA) is a novel imaging modality that visualises retinal microvasculature in a noninvasive manner. It may have value in managing retinopathy, correlate with visual outcome and mirror systemic involvement in autoimmune diseases.

Objectives: Aim of this study was to evaluate retinal microvasculature in systemic lupus erythematosus (SLE) patients and correlate abnormal vascular maps with disease activity, damage accrual, treatment and visual outcome.

Methods: In a prospective cross-sectional study, OCTA was used to examine density maps, by means of a $6 \mathrm{~mm}$ scan, based on superficial retinal layer and deeper retinal layer images in patients and healthy controls $(\mathrm{HC})$ (figure 1A-B) from November 2015 to December 2017. Vessel density, based on a map with vessels of 1-pixel width was measured.

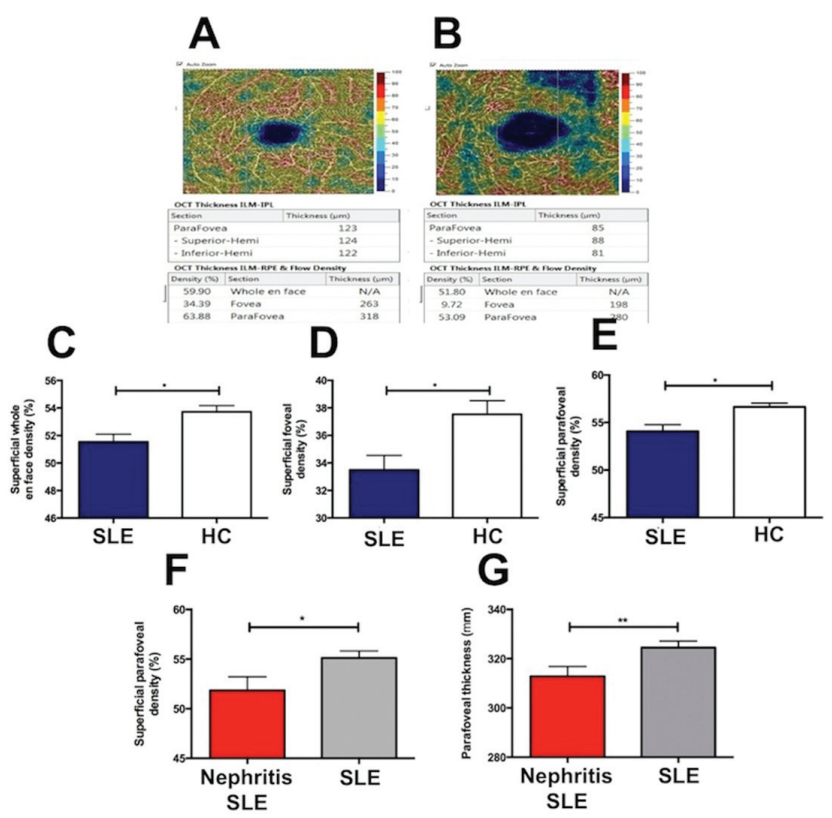

Results: 52 eyes from 26 SLE patients and 40 healthy eyes from $20 \mathrm{HC}$ were imaged (table 1). The eyes from SLE patients had a lower superficial whole en face density, superficial fovea density and superficial parafoveal density $(p=0.02$ for all comparisons) compared with healthy eyes (figure 1C-E). A negative correlation was demonstrated in SLE patients between age and superficial whole en face density ( $p=0.0005, r=-0.5)$, superficial foveal density $(p=0.006, r=-0.4)$, superficial parafoveal density $(p=0.004, r=-0.4)$, deep whole en face density $(p=0.003, r=-$ $0.4)$ and deep parafoveal density $(p=0.001, r=-0.4)$. SLEDAI correlated inversely with superficial en face density $(p=0.002, r=-0.4)$, superficial parafoveal density $(p=0.0003, r=-0.5$ and $p=0.002, r=-0.5)$, deep whole en face density $(p=0.01, r=-$ $0.4)$ and deep parafoveal density $(p=0.002, r=-0.4)$. A negative correlation was found between SLICC and superficial whole en face density $(p=0.0001, r=-0.5)$, superficial parafoveal density $(p<0.0001, r=-0.6)$, deep whole en face density $(p<0.0001, r=-0.6)$ and deep parafoveal density $(p<0.0001, r=-0.7)$. Patients with nephritis displayed reduced parafoveal vessel density and parafoveal thickness compared to those of patients without nephritis $(\mathrm{p}=0.02$ and $\mathrm{p}=0.008$, figure $1 \mathrm{~F}$ G). A positive correlation was found between hydroxychloroquine (HCQ) cumulative dose and both superficial and deep parafoveal density $(p=0.009, r=0.4$ and $\mathrm{p}=0.04, \mathrm{r}=0.3$ ). Best-corrected visual acuity in SLE positively correlated with superficial whole en face density, superficial parafoveal density, deep whole en face density, and deep parafoveal density ( $p<0.0001, r=0.7$ for all correlations). A negative correlation was found with fovea thickness $(p=0.01, r=-0.5)$.

Abstract OP0123 - Table 1

\begin{tabular}{lcc}
\hline & HC $(\mathbf{n}=\mathbf{2 0})$ & SLE $(\mathbf{n}=\mathbf{2 6})$ \\
\hline Age (years) & $46 \pm 8,9$ & $49,6 \pm 13,6$ \\
Female $n(\%)$ & $16(80)$ & $23(88.5)$ \\
Disease duration (months) & - & $181.5 \pm 93$ \\
SLEDAI & - & $4.3 \pm 4.2$ \\
SLICC & - & $1.9 \pm 1.5$ \\
HCQ cumulative dose (g) & - & $738.8 \pm 486.8$ \\
Kidney involvement (n/\%) & - & $10 / 40$ \\
NPSLE (n/\%) & & $8 / 32$ \\
aPL positive (n/\%) & - & $10 / 40$ \\
\hline
\end{tabular}

Conclusions: SLE patients, in particular those with kidney involvement, displayed a reduced retinal microvascular density compared with normal subjects Vessel density provides a quantitative metric of capillary network that correlated with age, best-corrected visual acuity and clinical features as SLE disease activity and damage accrual. Hydroxychloroquine might have a protective role preserving the microvascular structures.

Disclosure of Interest: None declared

DOI: 10.1136/annrheumdis-2018-eular.4276

\section{OP0124 $\quad$ IN SLE PATIENTS IN SUSTAINED LOW DISEASE ACTIVITY, NOVEL INTERFERON ASSAYS PREDICT FLARES AND GLUCOCORTICOID REQUIREMENTS}

K. Dutton, A. Psarras, Y. El-Sherbiny, M.Y. Md Yusof, P. Emery, E. Vital. Leeds Institute of Rheumatic and Musculoskeletal Medicine, University of Leeds, Leeds UK

Background: Objectives of therapy in SLE are to maintain low disease activity and minimise glucocorticoid exposure. Disease activity is unpredictable with periods of low disease activity followed by flares. Once disease is controlled, there is an unmet need for predictors of sustained remission or flares to decide when glucocorticoids can be safely tapered. Type 1 interferon (IFN-I) activity is associated with disease activity in SLE. We recently validated two novel assays for IFN-I. First, a 2-score gene expression system that is continuous and accounts for modularity of the IFN transcriptome. Second, the flow cytometric biomarker tetherin that allows measurement of IFN status in individual cell subsets, with memory $B$ cell tetherin (tetherin) correlating best with disease activity.

Objectives: To determine whether IFN assays can predict flare and glucocorticoid requirements in patients with lupus

Methods: Retrospective notes review was done in 165 consecutive patients with SLE who submitted IFN biomarker samples between 2011-2015. The reviewer was blinded to biomarker status.

For the interferon scores, RNA was extracted from PBMCs and a custom Taqman array was used to measure expression of 30 interferon stimulated genes normalised to $P P 1 A$ and then calculate IFN Score $A$ ( 12 genes predominantly responsive to IFN-alpha) and IFN Score B (14 genes also responsive to other IFN subtypes and inflammatory mediators). For tetherin, PBMCs were analysed fresh with con ventional surface staining. MFI of CD317 (tetherin) was measured on CD19+CD27+CD38 lymphocytes.

We performed two analyses: ${ }^{1}$ In patients in sustained low disease activity (defined as no BILAG A or B in the six months prior to IFN biomarker sampling), prediction of new disease activity in the following six months (defined as new BILAG A or B). The association between IFN biomarkers and change in mean monthly glucocorticoid dose following biomarker sampling (defined as same or increased vs decreased or no glucocorticoid prescription). Since tetherin is measured on Bcells we excluded patients who were B cell depleted after rituximab. IFN biomarkers were compared between groups using Mann-Whitney U Tests.

Results: Of 165 patients, 92 were in sustained low disease activity prior to biomarker sampling. Of these, new BILAG A/B activity occurred within 6 months of sampling in 16 (17\%). New BILAG A/B activity was associated with higher levels of IFN Score A $(p=0.027, n=83)$, IFN Score B $(p=0.097, n=83)$ and tetherin $(p=0.026, n=92)$. 